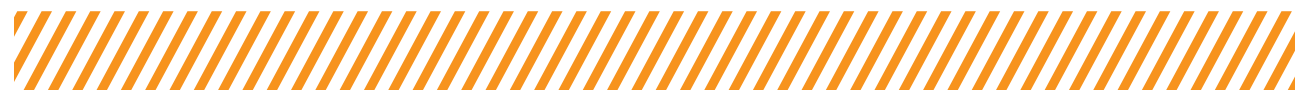

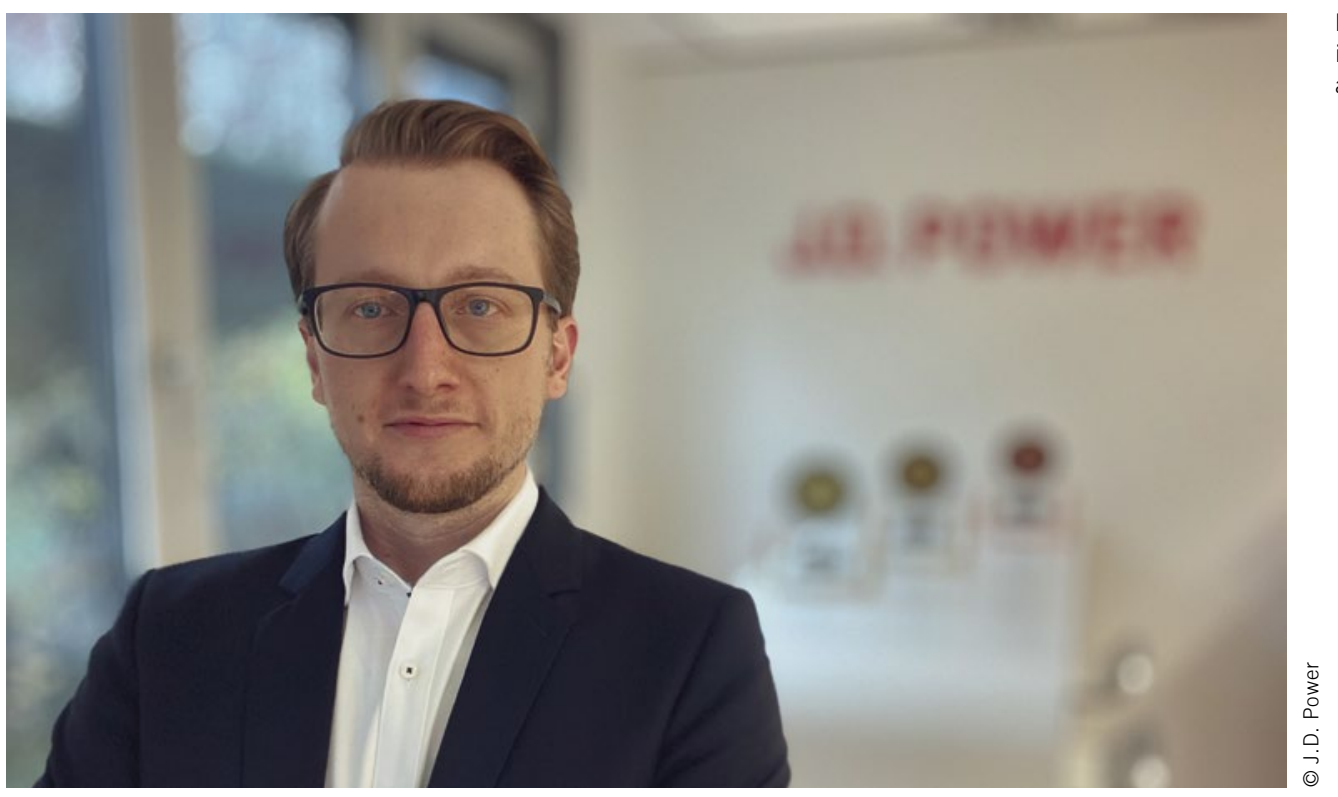

Fabian Chowanetz

is Manager Automotive Consulting at J.D. Power in Munich (Germany)

\section{OEMs Struggle to Deliver Intuitive Technology Experiences}

Automakers continue to face a gap between customer expectations and the ability of new technology to deliver a compelling experience. Owner satisfaction with infotainment, advanced driver assistance systems and smartphone apps connecting to the car remains among the least satisfying aspects of the vehicle experience.

As OEMs continue to add technology features that largely miss the mark in impressing drivers, this is bad news on two main fronts: First of all, a poor user experience leads to frustration that can diminish brand value and loyalty. This is a potential threat to current business that will only magnify as the industry moves toward more digitally driven products. Secondly, new technologies are expensive to develop, integrate and maintain. When they are underutilized or ignored, they contribute to cost without adding value for the customer.

Fixing this mismatch is an urgent priority for OEMs that want to maximize the value, appeal and profitability of their vehicles. It is especially important since hi-tech features are emerging as a primary factor in the vehicle purchase decision and user experience with features and services will be even more of a decision driver in the future. To address this challenge, automakers must develop better strategies for identifying and designing features that owners appreciate and find both intuitive and reliable.

OEMs need to consider initiatives that focus on a break-down of silos among design, engineering, quality and marketing. Different disciplines within their organizations are not aligned in their priorities and objectives for introducing new technology into vehicles. A joint focus must emerge for all relevant stakeholders based on a common understanding of customer desires, expectations and needs. Furthermore, there is a strong need to integrate customer centricity into corporate culture which is easier said than done. What is often missing is a follow through into enterprise-wide execution. There is opportunity to learn from digital industries that have mastered the ability to listen, understand and respond very specifically and quickly to the voice of the customer. Those manufacturers that put the customer at the center of how key groups are tasked, resourced and rewarded, will begin to close the gap.

Finally, consumer data needs to be integrated into the development process, quantitatively and qualitatively. While there has been much talk about the importance of data-driven decisionmaking in the automotive industry, many important decisions on innovation are still executed outside of this ideal. Listening to consumers early on allows OEMs to adjust to actual market demands before it becomes more difficult and expensive later in the process.

We have seen new disruptive players successfully establish a presence in the automotive space with the potential to take significant market share away from incumbent brands. Recognition of this threat is encouraging more industry leaders to get better at the "voice of the customer" imperative. Listening is a great first step. Now it's time for manufacturers to prove they can execute and deliver. 